\title{
Participant characteristics associated with greater reductions in waist circumference during a four- month, pedometer-based, workplace health program
}

Rosanne LA Freak-Poli*, Rory Wolfe, Helen Walls, Kathryn Backholer and Anna Peeters

\begin{abstract}
Background: Workplace health programs have demonstrated improvements in a number of risk factors for chronic disease. However, there has been little investigation of participant characteristics that may be associated with change in risk factors during such programs. The aim of this paper is to identify participant characteristics associated with improved waist circumference (WC) following participation in a four-month, pedometer-based, physical activity, workplace health program.

Methods: 762 adults employed in primarily sedentary occupations and voluntarily enrolled in a four-month workplace program aimed at increasing physical activity were recruited from ten Australian worksites in 2008. Seventy-nine percent returned at the end of the health program. Data included demographic, behavioural, anthropometric and biomedical measurements. WC change (before versus after) was assessed by multivariable linear and logistic regression analyses. Seven groupings of potential associated variables from baseline were sequentially added to build progressively larger regression models.
\end{abstract}

Results: Greater improvement in WC during the program was associated with having completed tertiary education, consuming two or less standard alcoholic beverages in one occasion in the twelve months prior to baseline, undertaking less baseline weekend sitting time and lower baseline total cholesterol. A greater WC at baseline was strongly associated with a greater improvement in WC. A sub-analysis in participants with a 'high-risk' baseline WC revealed that younger age, enrolling for reasons other than appearance, undertaking less weekend sitting time at baseline, eating two or more pieces of fruit per day at baseline, higher baseline physical functioning and lower baseline body mass index were associated with greater odds of moving to 'low risk' WC at the end of the program.

Conclusions: While employees with 'high-risk' WC at baseline experienced the greatest improvements in WC, the other variables associated with greater WC improvement were generally indicators of better baseline health. These results indicate that employees who started with better health, potentially due to lifestyle or recent behavioural changes, were more likely to respond positively to the program. Future health program initiators should think innovatively to encourage all enrolees along the health spectrum to achieve a successful outcome.

Keywords: waist circumference, workplace, association, prevention, risk-factor, cardiovascular disease, diabetes, health promotion, physical activity, pedometer

\footnotetext{
* Correspondence: Rosanne.Freak-Poli@monash.edu

'Department of Epidemiology \& Preventive Medicine, Faculty of Medicine, Nursing \& Health Sciences, School of Public Health and Preventive Medicine, Monash University, The Alfred Centre, Alfred Hospital, Commercial Road, Melbourne, Victoria 3004, Australia
} 


\section{Background}

Workplace health programs have demonstrated improvements in the leading global risk factors for chronic disease $[1,2]$ which has led to their increasing role in chronic disease prevention $[1,2]$. The majority of research has focused upon evaluating the program outcomes [1,3-7] and program characteristics $[2-5,7,8]$ which have increased the evidence-base for workplace health programs. However, there has been little evaluation of participant characteristics and process indicators that may be related to subsequent change in risk factors during such programs.

Identifying variables associated with a successful outcome in health promotion programs can help to determine the health program's reach and its effectiveness at enrolling a variety of participants, including employees with high health risks. The assessment of variables associated with the program can also be used to identify any inequities in the health program through identification of differences in outcome according to characteristics such as education status. Consequently, variables associated with program success can potentially be used to improve the reach and success of a health program, and this can be achieved by addressing any inequities in the targeting of enrolees or response to the program.

A number of workplace health program evaluations have demonstrated immediate improvements in physical activity, blood pressure and anthropometric measures [9]. As a direct intermediary between physical activity and disease [10-13], waist circumference (WC) can be considered a useful marker of the success of such programs. Only one workplace health program evaluation which considered participant characteristics associated with successful outcomes has been identified [14]. To adequately evaluate variables associated with success in workplace health programs, a comprehensive evaluation needs to be undertaken in a large workplace health program with a range of potential association variables including demographic, behavioural, anthropometric, biomedical and process measures, that can be (where appropriate) assessed in accordance to clinically relevant guidelines. We recently performed an evaluation of a four-month, pedometerbased, workplace health program, with a range of measures at baseline. A pre-post analysis of this program found that WC decreased on average by $1.6 \mathrm{~cm}[9,15]$.

The aim of this paper is to identify participant characteristics that are associated with greater improvements in waist circumference (WC) following participation in a four-month, pedometer-based, workplace health program.

\section{Methods}

\section{Study population}

Melbourne workplaces undertaking the 2008 Global Corporate Challenge ${ }^{\circledR}\left(G C^{\circledR}\right)$ event were approached to be evaluation sites. Following receipt of the Workplace
Consent, employees enrolled in the $2008 \mathrm{GCC}^{\circledR}$ event were approached via email. In early 2008, 762 eligible participants were recruited from ten workplaces, providing a variety of sedentary occupations $[9,15]$. Seventynine percent $(n=604)$ of participants returned directly after the health program for the four-month data collection [9]. Participants who returned for four-month data collection were less likely to report having diabetes and more likely to be older, participate in the $\mathrm{GCC}^{\circledR}$ due to health reasons, be a non-smoker and comply with the health program by undertaking 10,000 daily steps on average [9]. Eighty-eight percent $(n=671)$ of the total sample at baseline completed the WC measurement. Eighty percent $(n=539)$ of these participants returned to complete the WC measurement at four-months $(89 \%$ of the total sample who returned at four-months) [9].

\section{Description of the program}

The $\mathrm{GCC}^{\circledR}$ is the provider of a pedometer-based workplace program that is established world-wide and occurs annually. The program involves wearing a visible stepcount pedometer with a target of at least 10,000 steps per day for 125 days. Weekly encouragement emails are sent and a website is used for logging daily steps, accessing additional health information, communication amongst participants and comparing team progress. Participation requires an employer or employee financial contribution and is typically competitive.

\section{Data collection}

Data were collected directly prior to the $\mathrm{GCC}^{\circledR} 2008$ event (baseline) and immediately after completion of the GCC $^{\circledR} 2008$ event (four-month follow-up). In brief, trained staff visited employees' workplaces for scheduled morning appointments to collect fasting anthropometric and biomedical measurements. Before measurements, participants were asked to remove outer garments, belts and workplace ID tags from around their waists. To record WC, participants were asked to point out their lower rib margin and the top of the hip (iliac crest) and the measurement was taken midway. Waist was recorded using a Figure Finder Tape Measure (Novel Products Inc 2005 code PE024) and a mirror to ensure that the tape was horizontal. An Internet-based self-report questionnaire was completed by participants at their own convenience. The questionnaire incorporated demographic information [16-18], motivation and support for participation, a health history [16] and behavioural measures $[16,18,19]$. Meeting alcohol guidelines was defined as consuming two or fewer standard drinks on one occasion in the last twelve months [20]. Other national measurement guidelines for risk assessment are summarised in Table 1, including diabetes type 2 and cardiovascular disease predicted risk scores $[9,15]$. 


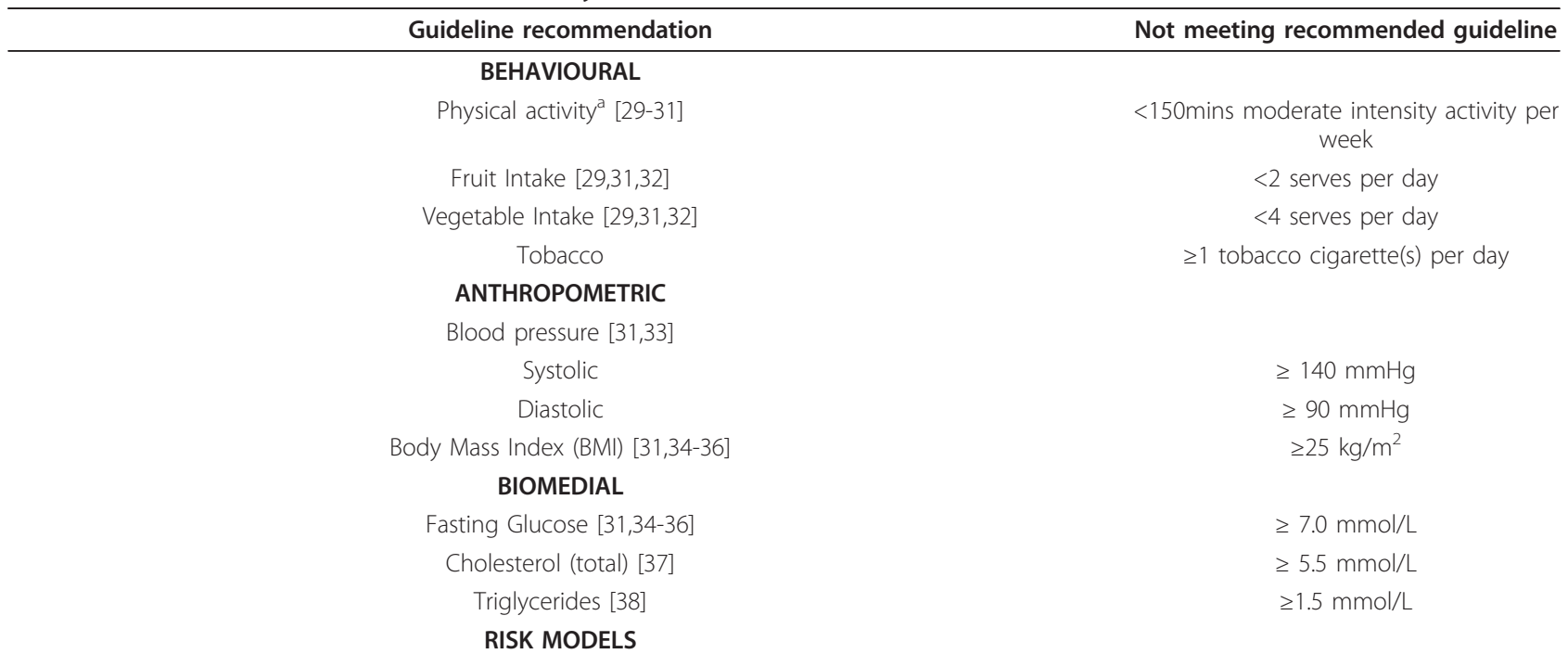

Diabetes type 2 5-year risk (assessed by The Australian Type 2 Diabetes Risk Assessment Tool - AUSDRISK) intermediate 1/100-1/20; high $\geq 1 / 20$ $[31,39,40]$

Cardiovascular disease (CVD) 10-year risk (assessed by the Absolute cardiovascular disease risk assessment intermediate 1/100-1/5; high $\geq 1 / 5$ tool) $[31,41-43]$

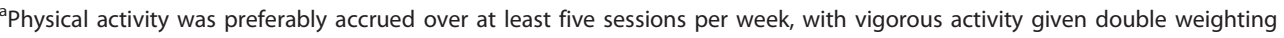

\section{Step information}

Step information was obtained by the GCC ${ }^{\circledR}$ through participation in the program. Participants were asked to enter their step-counts, as indicated on the pedometer, daily into the website diary. Bicycle ride length was also recorded on a daily basis and incorporated into the stepcount by the $\operatorname{GCC}^{\circledR}(6.4 \mathrm{~km}=10,000$ steps $)$.

Further methodological details of the $\mathrm{GCC}^{\circledR}$ Evaluation Study have been described elsewhere $[9,15]$.

\section{Outcome}

WC has been shown to be a better predictor of metabolic risk than body mass index, due to the independent association between an increased WC with health [10-13] and mortality [10,21-25]. Reduction in WC can be achieved through lifestyle changes and is an achievable goal for workplace health program initiatives. Recently it was identified that completion of this four-month, pedometer-based, workplace health program was associated with improvements in WC of $1.6 \mathrm{~cm}$ on average [9]. WC change for each participant was calculated by subtracting their baseline measurement from their four-month follow-up measurement.

\section{Variable selection}

All measured variables at baseline, i.e. participant characteristics present prior to commencing the program, were considered potential "predictors" of WC change in the sense that the characteristics pre-dated the programrelated changes. The only exception was the process variable, indicating compliance with the program, as measured by step count. Where possible these variables were considered as having a linear continuous-scale relationship with WC change rather than using categorisations that may be arbitrary. Several variables were assessed in accordance to clinically relevant guidelines as summarised in Table $1[9,15]$.

\section{Analysis}

All analyses were performed using Stata version 11 (Stata Corporation, TX). Robust standard errors, clustered by workplace, were used in all statistical analyses, including the calculation of confidence intervals. A p-value $<0.05$ was used to determine statistical significance.

Variables related to WC change were assessed by univariable and multivariable linear regression analyses with WC change as the outcome variable. This analysis was repeated in participants with high-risk WC, as defined in Table 1, at baseline that had also completed four-month data collection. Also in participants with high-risk WC at baseline, univariable and multivariable logistic regression analyses were fitted to high/low risk WC at follow-up.

To distinguish associated variables having an indirect influence on WC from participants exerting a more direct influence, two multiple regression approaches were taken. Potential association variables were formed into 10 separate and non-overlapping groups as follows.

1 Age (continuous), sex 
2 Socio economic status by SEIFA, tertiary education, occupation and marital status

3 Prior GCC $^{\circledR}$ Event participation and reasons for participation

4. Health behaviours

5 Psychosocial measures

6 Anthropometric measures

7 Biomedical measures

8 Predicted risk scores

9 Workplace characteristics

10 Step-data

In the first approach, the first two groups (baseline demographics) were used for adjustment in separate models containing one of the remaining 8 groups of variables (Multivariable Approach 1 in Tables 2, 3 and 4). In the second approach, these groups of variables were entered sequentially into the regression model, adjusting for the previous group as confounders (Multivariable Approach 2 in Tables 2, 3 and 4). Baseline WC was not included in the models in either approach due to its potential for introducing bias [26]. Consequently, as the diabetes type-2 predicted risk score included WC in the calculation, it was also excluded from the models. Instead, as described above, additional analyses were performed in a subset of participants identified as having high-risk baseline WC.

Glucose and triglyceride variables followed skewed distributions and were log-transformed before inclusion in regression models. Pregnant participants $(n=13)$ were excluded from analyses. Each analysis used participants with complete data on the relevant variable/s.

\section{Ethics}

The study, project number CF08/0271-2008000125, was approved by Monash University Human Research Ethics through the standing committee on ethics in researchinvolving humans.

\section{Results}

\section{Distribution of waist circumference change}

The change in waist circumference (WC) followed an approximately normal distribution with an average reduction of $1.6 \mathrm{~cm}( \pm 5.9 \mathrm{SD})$, Figure 1a. Males and females were similar in their mean WC reduction $(-1.3 \pm 5.5 \mathrm{SD}$ for males compared to $-1.8 \pm 6.2 \mathrm{SD}$ for females, $\mathrm{p}=0.4$ ), Figure 1b.

\section{Predictors of waist circumference improvement}

Between baseline and four-months, variables associated with WC improvement identified through univariable analyses included having completed tertiary education and less weekend or weekday sitting time at baseline, Table 2. Participants who were not meeting guidelines for WC at baseline (as defined in Table 1) responded

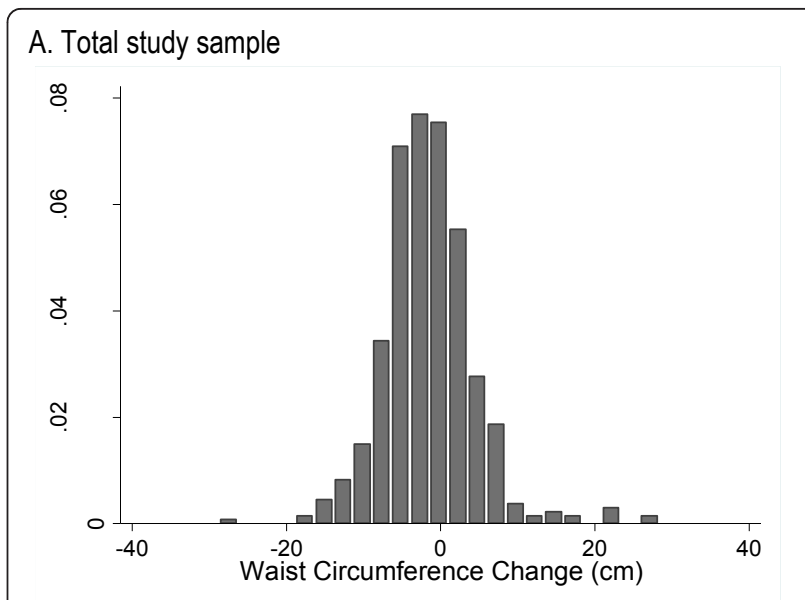

B. By sex

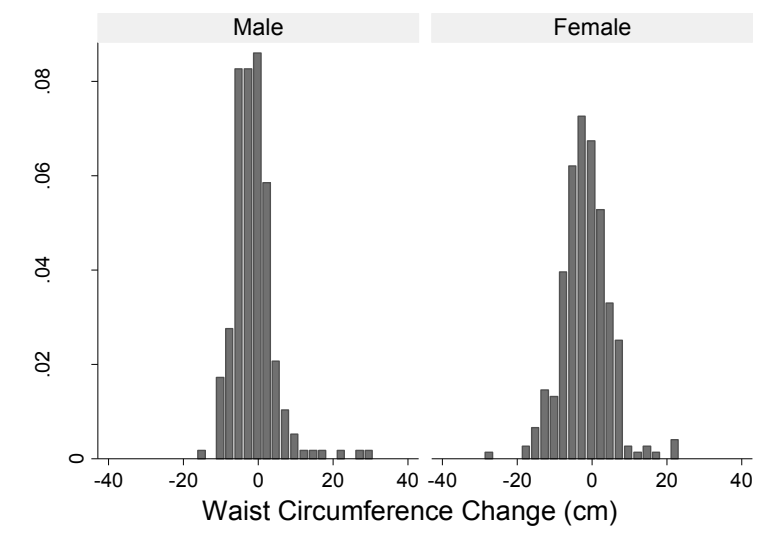

Figure 1 Distribution of waist circumference change associated with participation in a four-month workplace health program.

positively to the program and had a $2.9 \mathrm{~cm}$ larger reduction in WC than participants who were meeting guidelines for WC at baseline. For every extra centimetre of WC at baseline, a $0.12 \mathrm{~cm}$ loss in WC at four-months was observed. Similarly, participants with high baseline diabetes type- 2 risk had a $2.2 \mathrm{~cm}$ larger reduction in WC than participants with low baseline diabetes type-2 risk.

Multivariable approaches 1 and 2 produced similar results, Table 2. Between baseline and four-months, participants who had completed tertiary education at baseline had a $2.1 \mathrm{~cm}$ larger reduction in WC than participants who had not completed tertiary education at baseline. On average, participants who reported at baseline that they did not consume more than two alcoholic standard drinks in one occasion in the past twelve months reduced their WC by $0.7 \mathrm{~cm}$ more than participants who did not meet alcohol recommended guidelines. For every hour less per day of baseline weekend sitting time, a $0.3 \mathrm{~cm}$ greater loss in WC at four-months was observed. For every millimole per litre less of total cholesterol at baseline, a $0.4 \mathrm{~cm}$ greater loss in WC at four-months was observed. 
Table 2 Linear regression analyses assessing potential baseline and step-data predictors of waist circumference change

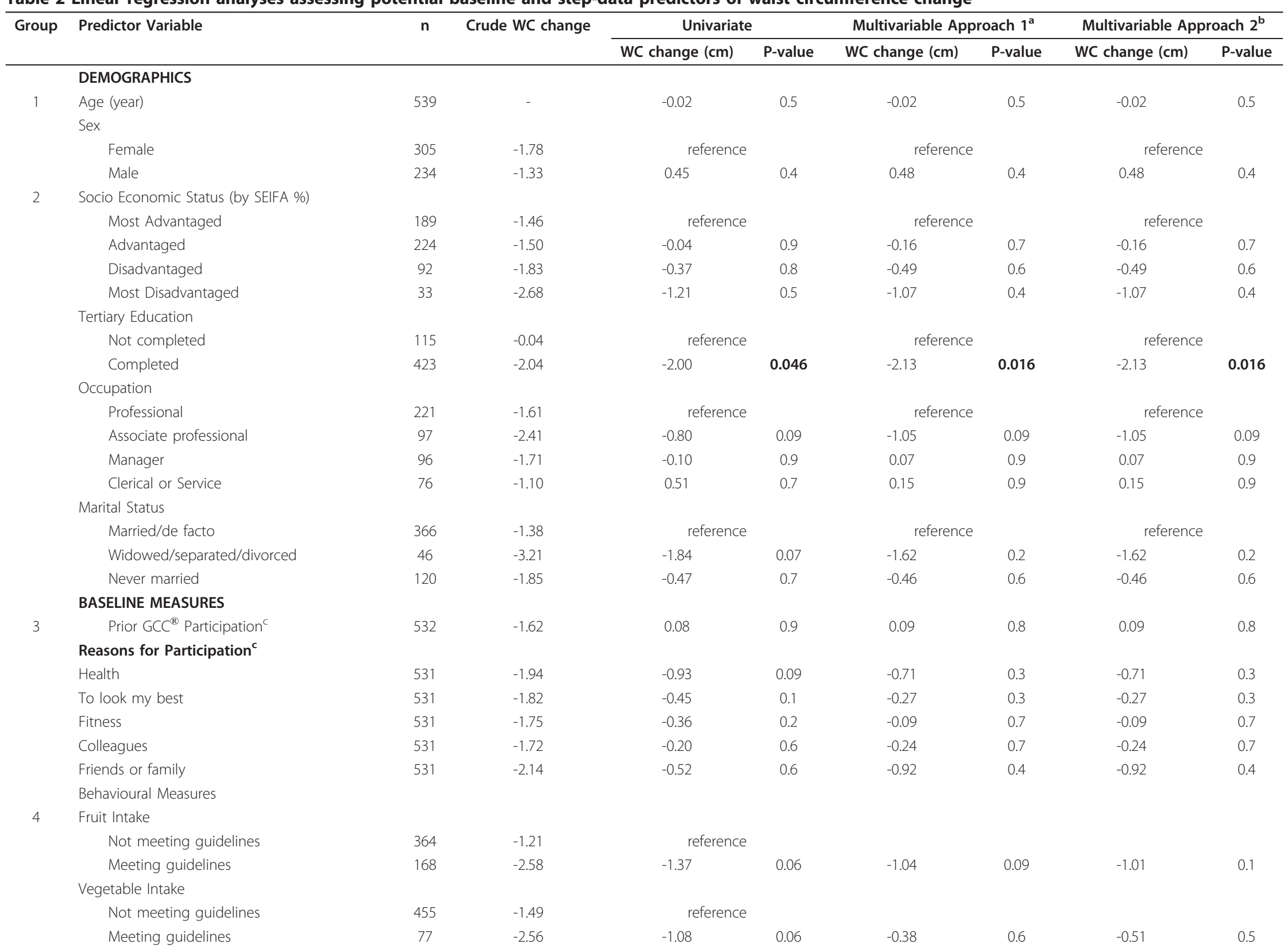


Table 2 Linear regression analyses assessing potential baseline and step-data predictors of waist circumference change (Continued)

\begin{tabular}{|c|c|c|c|c|c|c|c|c|c|}
\hline & Takeaway Dinner & & & & & & & & \\
\hline & Once or less per month & 246 & -1.68 & \multicolumn{2}{|c|}{ reference } & & & & \\
\hline & About once a week & 214 & -1.63 & 0.05 & 0.9 & -0.70 & 0.2 & -0.62 & 0.3 \\
\hline & More than once a week & 72 & -1.56 & 0.12 & 0.9 & -0.67 & 0.6 & -0.61 & 0.6 \\
\hline & \multicolumn{9}{|l|}{ Alcohol } \\
\hline & Not meeting guidelines & 307 & -1.42 & \multicolumn{2}{|c|}{ reference } & & & & \\
\hline & Meeting guidelines & 225 & -1.95 & -0.54 & 0.2 & -0.58 & 0.035 & -0.65 & 0.016 \\
\hline & \multicolumn{9}{|l|}{ Tobacco } \\
\hline & Smoker & 50 & -1.00 & \multicolumn{2}{|c|}{ reference } & & & & \\
\hline & Non-smoker & 482 & -1.71 & -0.71 & 0.4 & 0.13 & 0.9 & 0.32 & 0.7 \\
\hline & \multicolumn{9}{|l|}{ Physical Activity } \\
\hline & Not meeting guidelines & 322 & -1.49 & \multicolumn{2}{|c|}{ reference } & & & & \\
\hline & Meeting guidelines & 209 & -1.87 & -0.38 & 0.5 & 0.00 & 1.0 & -0.10 & 0.9 \\
\hline & \multicolumn{9}{|l|}{ Sitting Time (per hour per day) } \\
\hline & Weekday & 530 & - & 0.19 & 0.015 & 0.09 & 0.048 & 0.10 & 0.06 \\
\hline & Weekend & 529 & - & 0.12 & 0.026 & 0.28 & 0.018 & 0.28 & 0.025 \\
\hline & \multicolumn{9}{|l|}{ Psychosocial Measures } \\
\hline \multirow[t]{3}{*}{5} & Physical Functioning (SF12) & 523 & - & -0.06 & 0.09 & -0.05 & 0.08 & -0.05 & 0.2 \\
\hline & Mental Functioning (SF12) & 523 & - & 0.00 & 0.9 & -0.02 & 0.6 & -0.01 & 0.5 \\
\hline & \multicolumn{9}{|l|}{ Anthropometric Measures ${ }^{d}$} \\
\hline \multirow[t]{16}{*}{6} & \multicolumn{9}{|l|}{ Blood Pressure $(\mathrm{mmHg})$} \\
\hline & Systolic & 517 & - & -0.01 & 0.5 & 0.00 & 1.0 & 0.00 & 0.9 \\
\hline & Diastolic & 517 & - & 0.03 & 0.2 & -0.03 & 0.6 & -0.03 & 0.5 \\
\hline & Not meeting guidelines & 90 & -1.72 & \multicolumn{2}{|c|}{ reference } & & & & \\
\hline & Meeting guidelines & 427 & -1.51 & 0.20 & 0.6 & & & & \\
\hline & Heart rate (Mean, SD) & 517 & - & -0.03 & 0.3 & -0.02 & 0.5 & -0.04 & 0.2 \\
\hline & Weight (Mean, SD) & 537 & - & -0.02 & 0.4 & & & & \\
\hline & \multicolumn{9}{|l|}{ Body Mass Index } \\
\hline & Body Mass Index & 222 & - & -0.07 & 0.08 & -0.08 & 0.08 & -0.08 & 0.08 \\
\hline & Not meeting guidelines & 315 & -1.68 & \multicolumn{2}{|c|}{ reference } & & & & \\
\hline & Meeting guidelines & 222 & -1.51 & 0.17 & 0.8 & & & & \\
\hline & \multicolumn{9}{|l|}{ Waist circumference ${ }^{f}$} \\
\hline & Waist circumference & 539 & - & -0.12 & 0.001 & & & & \\
\hline & Not meeting guidelines & 288 & -2.86 & \multicolumn{2}{|c|}{ reference } & & & & \\
\hline & Meeting guidelines & 251 & -0.12 & 2.74 & 0.001 & & & & \\
\hline & Biomedical Measures (fasting) $^{d}$ & & & & & & & & \\
\hline \multirow[t]{3}{*}{7} & Total Cholesterol & & & & & & & & \\
\hline & Total Cholesterol & 535 & - & 0.20 & 0.4 & 0.49 & 0.034 & 0.38 & 0.029 \\
\hline & Not meeting guidelines & 150 & -1.66 & \multicolumn{2}{|c|}{ reference } & & & & \\
\hline
\end{tabular}

ting Time (per hour per day)

reference 
Table 2 Linear regression analyses assessing potential baseline and step-data predictors of waist circumference change (Continued)

\begin{tabular}{|c|c|c|c|c|c|c|c|c|c|}
\hline & Meeting guidelines & 385 & -1.63 & 0.03 & 1.0 & & & & \\
\hline \multicolumn{10}{|c|}{$\begin{array}{l}\text { Mleeting guidellnes } \\
\text { Glucose }\end{array}$} \\
\hline & Glucose & 535 & - & 0.12 & 0.3 & 0.06 & 0.2 & 0.02 & 0.09 \\
\hline & Not meeting guidelines & 20 & 0.05 & \multicolumn{2}{|c|}{ reference } & & & & \\
\hline & Meeting guidelines & 535 & 1.49 & 4.23 & 0.3 & & & & \\
\hline & \multicolumn{9}{|l|}{ Triglycerides } \\
\hline & Triglycerides & 535 & - & 0.83 & 0.7 & 0.49 & 0.1 & 0.99 & 1.0 \\
\hline & Not meeting guidelines & 109 & 0.10 & \multicolumn{2}{|c|}{ reference } & & & & \\
\hline & Meeting guidelines & 426 & 2.43 & 2.33 & 0.1 & & & & \\
\hline & \multicolumn{9}{|l|}{ Predicted risk scores $^{\mathrm{d}}$} \\
\hline \multirow[t]{11}{*}{8} & \multicolumn{9}{|l|}{ Cardiovascular disease risk (next 10 years) } \\
\hline & CVD risk (continuous) & 504 & - & -0.03 & 0.6 & -0.08 & 0.2 & -0.11 & 0.4 \\
\hline & Low-risk & 452 & -1.61 & \multicolumn{2}{|c|}{ reference } & & & & \\
\hline & Intermediate-risk & 45 & -1.76 & -0.14 & 0.9 & & & & \\
\hline & High-risk & 7 & -2.91 & -1.30 & 0.056 & & & & \\
\hline & \multicolumn{9}{|l|}{ Diabetes risk (next 5 years) ${ }^{f}$} \\
\hline & Diabetes risk (continuous) & 529 & - & -0.09 & 0.09 & & & & \\
\hline & Low-risk & 202 & -1.26 & \multicolumn{2}{|c|}{ reference } & & & & \\
\hline & Intermediate-risk & 276 & -1.55 & -0.28 & 0.6 & & & & \\
\hline & High-risk & 51 & -3.45 & -2.19 & 0.004 & & & & \\
\hline & \multicolumn{9}{|l|}{ WORKPLACE CHARACTERISTICS ${ }^{g}$} \\
\hline \multirow[t]{4}{*}{9} & Public ownership (vs. private) & 539 & -2.00 & -0.67 & 0.4 & -0.57 & 0.2 & -0.37 & 0.5 \\
\hline & Outer city location (vs. inner city) & 539 & -2.22 & -0.37 & 0.7 & 0.16 & 0.8 & 0.06 & 1.0 \\
\hline & \multicolumn{9}{|l|}{ PROCESS MEASURES } \\
\hline & \multicolumn{9}{|l|}{ Step data ${ }^{d}$} \\
\hline \multirow[t]{3}{*}{10} & Step average per day (per 10,000 steps) & 538 & - & -0.20 & 0.7 & -0.25 & 0.8 & 0.12 & 0.9 \\
\hline & $<10,000$ steps average (per day) & 179 & -1.48 & reference & & & & & \\
\hline & Meeting $\geq 10,000$ steps average (per day) & 359 & -1.65 & -0.17 & 0.7 & & & & \\
\hline
\end{tabular}

${ }^{a}$ Associated risk-factor subgroups adjusted only for baseline demographic confounders

${ }^{\mathrm{b}}$ Associated risk-factor subgroups adjusted for all potential predictors within the analysis step and above

' The reference group for this binary variable is 'no'. The reference group data is not shown.

${ }^{d}$ Continuous variables chosen over categorical variables

excluded due to the inclusion of body mass index (BMI)

${ }^{f}$ Excluded from model as baseline WC was not considered as a predictor.

${ }^{g}$ The reference group for this binary variable is shown in brackets. The reference group data is not shown. 
Table 3 Comparison of baseline characteristics between high-risk and low-risk waist circumference at baseline $e_{a}$

\section{Low-risk WC \\ (Mean \pm SD or Percentage)}

\section{WORKPLACE CHARACTERISTICS}

Public ownership (vs. private)

251

High-risk WC
(Mean \pm SD or Percentage)

288

Inner city location (vs. outer city)

DEMOGRAPHICS

Age (year)

Male

Socio Economic Status (by SEIFA)

$$
\begin{array}{r}
\text { Most Advantaged } \\
\text { Advantaged } \\
\text { Disadvantaged } \\
\text { Most Disadvantaged }
\end{array}
$$

Completion of tertiary education

Occupation

$$
\begin{array}{r}
\text { Professional } \\
\text { Associate professional } \\
\text { Manager } \\
\text { Clerical or Service }
\end{array}
$$

Marital Status

$$
\text { Married or de facto }
$$

Widowed, separated or divorced

Never married

\section{BASELINE MEASURES}

Prior GCC ${ }^{\circledR}$ Participation ${ }^{C}$

Motivation for Participation

Health

To look my best

Fitness

Colleagues

Friends or family

\section{Behavioural Measures}

Self reported hypertension

Self reported diabetes

Fruit Intake (meeting guidelines)

Vegetable Intake (meeting guidelines)

Takeaway Dinner

$$
\begin{array}{r}
\text { Once or less per month } \\
\text { About once a week } \\
\text { More than once a week }
\end{array}
$$

Alcohol (meeting guidelines)

Non tobacco smoker

Physical Activity (meeting guidelines)

Sitting Time (hrs per day)

$$
\text { Weekday }
$$$$
\text { Weekend }
$$

\section{Physical Measures}

Systolic Blood Pressure $(\mathrm{mmHg})$

Diastolic Blood Pressure $(\mathrm{mmHg})$

Blood Pressure (meeting guidelines)

Heart rate (beats per minute)

Weight (kg)

\section{7}

70.9

$38 \pm 10$

52.2

37.5

40.6

15.9

6.0

81.6

50.7

19.2

17.9

12.2

67.5

8.1

24.4

21.54

56.7

43.3

60.8

58.4

2.5

10.3

2.1

28.5

14.2

46.3

39.4

14.2

39.8

92.7

41.2

$8.3 \pm 3.6$

$5.7 \pm 3.1$

$116.1 \pm 14.0$

86.1

$67.3 \pm 10.2$

$68.7 \pm 11.1$
$77.2 \pm 10.0$
43.1

67.7

$43 \pm 10$

35.8

33.1

42.5

18.1

6.3

76.0

0.2

40.2

20.3

21.1

18.4

69.9

9.1

21.0

24.83

0.06

76.6

$<0.001$

72.7

$<0.001$

73.1

0.038

0.4

1.0

2.5

0.001

0.001

0.5

0.9

0.9

0.1

0.2

0.018

37.8

0.8

$8.2 \pm 3.6$

0.001

$120.9 \pm 15.0$

0.010

$81.8 \pm 10.1$

0.001

79.5

0.036

$69.5 \pm 9.3$

0.007

$84.1 \pm 15.4$ 
Table 3 Comparison of baseline characteristics between high-risk and low-risk waist circumference at baseline (Continued)

\begin{tabular}{|c|c|c|c|}
\hline Body Mass Index $\left(\mathrm{kg} / \mathrm{m}^{2}\right)$ & $23.6 \pm 2.4$ & $29.2 \pm 4.5$ & $<0.001$ \\
\hline Body Mass Index (meeting guidelines) & 69.1 & 17.4 & $<0.001$ \\
\hline Waist circumference $(\mathrm{cm})$ & $79.4 \pm 8.1$ & $95.2 \pm 10.2$ & $<0.001$ \\
\hline Waist circumference (meeting guidelines) & 100.0 & 0.0 & $<0.001$ \\
\hline \multicolumn{4}{|l|}{ Biomedical Measures (fasting) } \\
\hline Total Cholesterol (mmol/L) & $4.8 \pm 0.9$ & $5.0 \pm 1.0$ & 0.1 \\
\hline Total Cholesterol (meeting guidelines) & 75.6 & 68.8 & 0.025 \\
\hline Glucose (mmol/L) & $4.9 \pm 0.7$ & $5.2 \pm 1.1$ & 0.004 \\
\hline Glucose (meeting guidelines) & 99.2 & 93.7 & 0.025 \\
\hline Triglycerides (mmol/L) & $1.0 \pm 0.5$ & $1.3 \pm 1.0$ & 0.003 \\
\hline Triglycerides (meeting guidelines) & 85.6 & 74.4 & $<0.001$ \\
\hline \multicolumn{4}{|l|}{ Predicted risk scores } \\
\hline \multicolumn{4}{|l|}{ Cardiovascular disease risk (next 10 years) } \\
\hline CVD risk & $2.9 \pm 4.2$ & $5.5 \pm 5.2$ & 0.001 \\
\hline High-risk & 0.00 & 2.62 & 0.002 \\
\hline Intermediate-risk & 5.06 & 12.36 & \\
\hline \multicolumn{4}{|l|}{ Diabetes risk (next 5 years) } \\
\hline Diabetes risk & $5.5 \pm 3.3$ & $9.3 \pm 5.1$ & $<0.001$ \\
\hline High-risk & 2.5 & 15.8 & $<0.001$ \\
\hline Intermediate-risk & 42.2 & 60.7 & \\
\hline \multicolumn{4}{|l|}{ PROCESS MEASURES } \\
\hline \multicolumn{4}{|l|}{ Step data } \\
\hline Steps average (per day) & $11815 \pm 3661$ & $11491 \pm 3690$ & 0.5 \\
\hline Meeting 10,000 steps average (per day) & 67.6 & 66.0 & 0.8 \\
\hline \multicolumn{4}{|l|}{ FOUR-MONTH MEASURES } \\
\hline Waist circumference $(\mathrm{cm})$ & $79.3 \pm 8.8$ & $92.3 \pm 11.32$ & $<0.001$ \\
\hline Waist circumference (meeting guidelines) & 91.2 & 22.9 & $<0.001$ \\
\hline
\end{tabular}

High-risk versus low-risk baseline waist circumference To investigate whether variables associated with WC change alter for participants most at risk, further analyses were undertaken in this sub-group. Fifty-three percent $(\mathrm{n}=288)$ of participants were categorised as having a high-risk WC at baseline. Participants who had a high-risk WC at baseline were more likely to be older; be female; have a managerial, clerical or service occupation; work for a publically owned company; participate in the program due to health, fitness or appearance reasons; and have poorer health indicators when compared to baseline low-risk WC participants who returned at four-months, Table 3.

\section{Predictors of continuous waist circumference improvement in participants with high-risk waist circumference at baseline}

Within the baseline high-risk WC group, variables associated with WC improvement identified through univariable analyses included having completed tertiary education, eating two or more serves of fruit per day, having greater physical functioning at baseline and meeting the goal of at least 10,000 steps per day on average during the health program, Table 4. Within the high WC risk baseline group, a weak association between baseline WC and improved WC was observed, however it was not statistically significant nor was it of the same magnitude of the observed association in all participants. An additional analysis within the high-risk WC group comparing the BMI in the obese group to the BMI in the normal group in regards to WC change indicated weak evidence of a relationship $(2.8 \mathrm{~cm}$ greater loss in the normal weight group, $\mathrm{p}=0.06$ ). Participants with moderate baseline diabetes type- 2 risk had a $1.4 \mathrm{~cm}$ larger reduction in WC when compared to participants with low baseline diabetes type- 2 risk, however no relationship with high diabetes type- 2 risk was identified.

In participants who had a high-risk WC at baseline, participants who had completed tertiary education at baseline had a $2.4 \mathrm{~cm}$ larger reduction in $\mathrm{WC}$ when 
Table 4 Linear regression assessing baseline ${ }^{a}$ variables associated with waist circumference change in participants not meeting WC guidelines

\begin{tabular}{|c|c|c|c|c|c|c|c|c|c|}
\hline \multirow[t]{2}{*}{ Group } & \multirow[t]{2}{*}{ Predictor Variable } & \multirow[t]{2}{*}{$\mathrm{n}$} & \multirow[t]{2}{*}{ Crude WC change } & \multicolumn{2}{|l|}{ Univariate } & \multicolumn{2}{|c|}{ Multivariable Approach $1^{\text {a }}$} & \multicolumn{2}{|c|}{ Multivariable Approach $2^{\mathrm{b}}$} \\
\hline & & & & WC change $(\mathrm{cm})$ & P-value & WC change $(\mathrm{cm})$ & P-value & WC change $(\mathrm{cm})$ & P-value \\
\hline \multicolumn{10}{|c|}{ DEMOGRAPHICS } \\
\hline \multirow[t]{4}{*}{1} & Age (year) & 288 & - & 0.03 & 0.4 & 0.03 & 0.4 & 0.03 & 0.4 \\
\hline & Sex & & & & & & & & \\
\hline & Female & 185 & -2.94 & \multicolumn{2}{|l|}{ reference } & \multicolumn{2}{|c|}{ reference } & \multicolumn{2}{|c|}{ reference } \\
\hline & Male & 103 & -2.73 & 0.21 & 0.6 & 0.16 & 0.7 & 0.16 & 0.7 \\
\hline \multirow[t]{18}{*}{2} & Socio Economic Status (by SEIFA \%) & & & & & & & & \\
\hline & Most Advantaged & 95 & -2.84 & \multicolumn{2}{|l|}{ reference } & \multicolumn{2}{|c|}{ reference } & \multicolumn{2}{|c|}{ reference } \\
\hline & Advantaged & 122 & -2.61 & 0.23 & 0.8 & 0.32 & 0.6 & 0.32 & 0.6 \\
\hline & Disadvantaged & 52 & -2.82 & 0.02 & 1.0 & 0.31 & 0.9 & 0.31 & 0.9 \\
\hline & Most Disadvantaged & 18 & -5.78 & -2.94 & 0.1 & -2.11 & 0.3 & -2.11 & 0.3 \\
\hline & Tertiary Education & & & & & & & & \\
\hline & Not completed & 69 & -1.08 & \multicolumn{2}{|l|}{ reference } & \multicolumn{2}{|c|}{ reference } & \multicolumn{2}{|c|}{ reference } \\
\hline & Completed & 219 & -3.43 & -2.35 & 0.018 & -2.35 & 0.017 & -2.35 & 0.017 \\
\hline & \multicolumn{9}{|l|}{ Occupation } \\
\hline & Professional & 105 & -2.34 & \multicolumn{2}{|l|}{ reference } & \multicolumn{2}{|c|}{ reference } & \multicolumn{2}{|c|}{ reference } \\
\hline & Associate professional & 53 & -4.13 & -1.79 & 0.06 & -1.89 & 0.1 & -1.89 & 0.1 \\
\hline & Manager & 55 & -3.52 & -1.18 & 0.08 & -1.00 & 0.2 & -1.00 & 0.2 \\
\hline & Clerical or Service & 48 & -2.84 & -0.50 & 0.7 & -1.06 & 0.4 & -1.06 & 0.4 \\
\hline & \multicolumn{9}{|l|}{ Marital Status } \\
\hline & Married/de facto & 200 & -2.45 & reference & & referenc & & referenc & \\
\hline & Widowed/separated/divorced & 26 & -4.82 & -2.37 & 0.2 & -2.42 & 0.2 & -2.42 & 0.2 \\
\hline & Never married & 60 & -3.48 & -1.02 & 0.3 & -0.36 & 0.6 & -0.36 & 0.6 \\
\hline & BASELINE MEASURES & & & & & & & & \\
\hline 3 & Prior GCC ${ }^{\circledR}$ Participation ${ }^{\complement}$ & 286 & -2.86 & 0.08 & 0.8 & -0.04 & 0.9 & -0.04 & 0.9 \\
\hline & Reasons for Participation ${ }^{c}$ & & & & & & & & \\
\hline & Health & 286 & -2.82 & 0.27 & 0.8 & 0.14 & 0.9 & 0.14 & 0.9 \\
\hline & To look my best & 286 & -2.80 & 0.32 & 0.5 & 0.32 & 0.6 & 0.32 & 0.6 \\
\hline & Fitness & 286 & -2.75 & 0.49 & 0.4 & 0.07 & 0.9 & 0.07 & 0.9 \\
\hline & Colleagues & 286 & -3.24 & -0.81 & 0.053 & -0.72 & 0.3 & -0.72 & 0.3 \\
\hline & Friends or family & 286 & -4.01 & -1.15 & 0.2 & -1.10 & 0.3 & -1.10 & 0.3 \\
\hline & Behavioural Measures & & & & & & & & \\
\hline 4 & Fruit Intake & & & & & & & & \\
\hline & Not meeting guidelines & 188 & -2.42 & reference & & & & & \\
\hline & Meeting guidelines & 98 & -3.77 & -1.34 & 0.027 & -0.96 & 0.3 & -0.96 & 0.3 \\
\hline & Vegetable Intake & & & & & & & & \\
\hline & Not meeting guidelines & 244 & -2.77 & reference & & & & & \\
\hline & Meeting guidelines & 42 & -3.52 & -0.75 & 0.4 & 0.40 & 0.8 & 0.32 & 0.8 \\
\hline
\end{tabular}


Table 4 Linear regression assessing baseline ${ }^{a}$ variables associated with waist circumference change in participants not meeting WC guidelines (Continued)

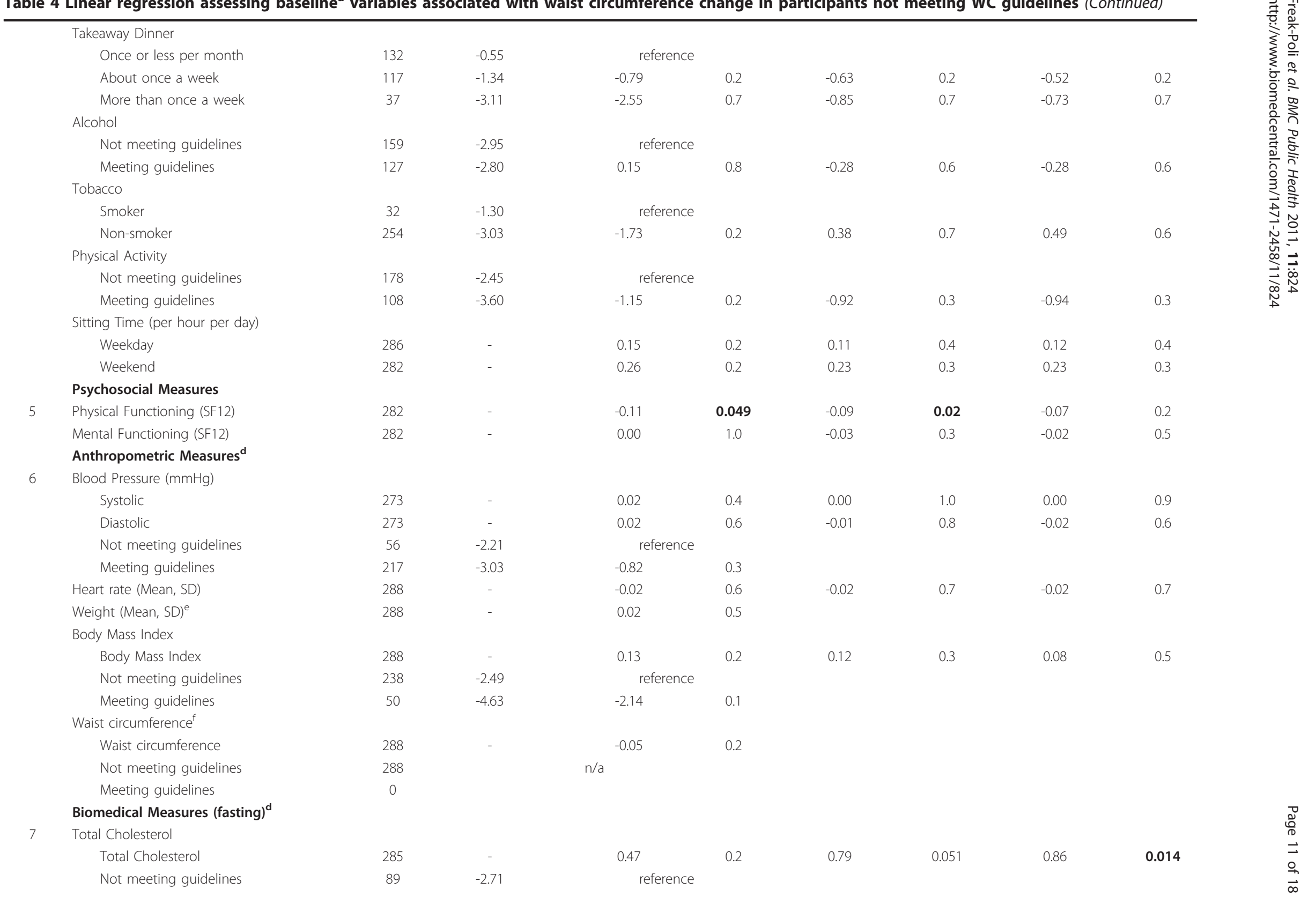


Table 4 Linear regression assessing baseline ${ }^{a}$ variables associated with waist circumference change in participants not meeting WC guidelines (Continued)

\begin{tabular}{|c|c|c|c|c|c|c|c|c|c|}
\hline & Meeting guidelines & 196 & -2.94 & -0.23 & 0.7 & & & & \\
\hline & \multicolumn{9}{|l|}{ Glucose } \\
\hline & Glucose & 285 & - & 2.29 & 0.8 & 0.22 & 0.6 & 0.03 & 0.3 \\
\hline & Not meeting guidelines & 18 & 0.06 & \multicolumn{2}{|c|}{ reference } & & & & \\
\hline & Meeting guidelines & 267 & 1.07 & 1.02 & 1.0 & & & & \\
\hline & \multicolumn{9}{|l|}{ Triglycerides } \\
\hline & Triglycerides & 285 & - & 1.31 & 0.6 & 0.47 & 0.2 & 0.45 & 0.2 \\
\hline & Not meeting guidelines & 73 & 0.03 & \multicolumn{2}{|c|}{ reference } & & & & \\
\hline & Meeting guidelines & 212 & 1.96 & 1.93 & 0.4 & & & & \\
\hline & Predicted risk scores $^{\mathrm{d}}$ & & & & & & & & \\
\hline \multirow[t]{11}{*}{8} & Cardiovascular disease risk (next 10 years) & & & & & & & & \\
\hline & CVD risk (continuous) & 267 & - & 0.09 & 0.2 & 0.03 & 0.7 & 0.02 & 0.9 \\
\hline & Low-risk & 227 & -2.89 & \multicolumn{2}{|c|}{ reference } & & & & \\
\hline & Intermediate-risk & 33 & -2.79 & 0.09 & 0.9 & & & & \\
\hline & High-risk & 7 & -2.91 & -0.03 & 1.0 & & & & \\
\hline & \multicolumn{9}{|l|}{ Diabetes risk (next 5 years) ${ }^{\mathrm{f}}$} \\
\hline & Diabetes risk (continuous) & 285 & - & 0.06 & 0.3 & & & & \\
\hline & Low-risk & 67 & -3.79 & \multicolumn{2}{|c|}{ reference } & & & & \\
\hline & Intermediate-risk & 173 & -2.34 & 1.45 & 0.033 & & & & \\
\hline & High-risk & 45 & -3.55 & 0.24 & 0.6 & & & & \\
\hline & WORKPLACE CHARACTERISTICS ${ }^{g}$ & & & & & & & & \\
\hline \multirow[t]{4}{*}{9} & Public ownership (vs. private) & 288 & -2.66 & 0.36 & 0.6 & 0.09 & 0.9 & 0.06 & 0.9 \\
\hline & Outer city location (vs. inner city) & 288 & -3.08 & -0.68 & 0.4 & -0.96 & 0.3 & -1.34 & 0.2 \\
\hline & PROCESS MEASURES & & & & & & & & \\
\hline & Step data ${ }^{d}$ & & & & & & & & \\
\hline \multirow[t]{3}{*}{10} & Step average per day (per 10,000 steps) & 288 & - & -1.04 & 0.08 & -1.40 & 0.07 & -0.55 & 0.6 \\
\hline & $<10,000$ steps average (per day) & 190 & -2.13 & \multicolumn{2}{|c|}{ reference } & & & & \\
\hline & Meeting $\geq 10,000$ steps average (per day) & 190 & -3.24 & -1.11 & 0.049 & & & & \\
\hline
\end{tabular}

${ }^{a}$ Associated risk-factor subgroups adjusted only for baseline demographic confounders

${ }^{b}$ Associated risk-factor subgroups adjusted for all potential predictors within the analysis Group and above

' The reference group for this binary variable is 'no'. The reference group data is not shown.

${ }^{d}$ Continuous variables chosen over categorical variables

${ }^{e}$ Excluded due to the inclusion of body mass index (BMI)

${ }^{\mathrm{f}}$ Excluded from model as baseline WC was not considered as a predictor.

${ }^{g}$ The reference group for this binary variable is shown in brackets. The reference group data is not shown.

Note: For participants not meeting WC guidelines at baseline 


\begin{tabular}{|c|c|c|c|c|c|c|c|c|c|}
\hline \multirow[t]{2}{*}{ Group } & \multirow[t]{2}{*}{ Predictor Variable } & \multirow[t]{2}{*}{$n$} & \multirow[t]{2}{*}{$\%$ meeting WC guidelines at four-months } & \multicolumn{2}{|c|}{ Univariate } & \multicolumn{2}{|c|}{ Multivariable Approach $1^{a}$} & \multicolumn{2}{|c|}{ Multivariable Approach 2} \\
\hline & & & & OR & P-value & OR & P-value & OR & P-value \\
\hline & DEMOGRAPHICS & & & & & & & & \\
\hline \multirow[t]{4}{*}{1} & Age (year) & 288 & - & 0.97 & 0.08 & 0.97 & 0.039 & 0.97 & 0.039 \\
\hline & Sex & & & & & & & & \\
\hline & Female & 185 & 21.08 & \multicolumn{2}{|c|}{ reference } & \multicolumn{2}{|c|}{ reference } & \multicolumn{2}{|c|}{ reference } \\
\hline & Male & 103 & 26.21 & 1.33 & 0.4 & 1.43 & 0.3 & 1.43 & 0.3 \\
\hline \multirow[t]{18}{*}{2} & Socio Economic Status (by SEIFA \%) & & & & & & & & \\
\hline & Most Advantaged & 95 & 21.05 & \multicolumn{2}{|c|}{ reference } & \multicolumn{2}{|c|}{ reference } & \multicolumn{2}{|c|}{ reference } \\
\hline & Advantaged & 122 & 22.13 & 1.07 & 0.9 & 1.03 & 0.9 & 1.03 & 0.9 \\
\hline & Disadvantaged & 52 & 26.92 & 1.38 & 0.6 & 1.39 & 0.5 & 1.39 & 0.5 \\
\hline & Most Disadvantaged & 18 & 27.78 & 1.44 & 0.4 & 1.63 & 0.2 & 1.63 & 0.2 \\
\hline & Tertiary Education & & & & & & & & \\
\hline & Not completed & 69 & 20.29 & \multicolumn{2}{|c|}{ reference } & \multicolumn{2}{|c|}{ reference } & \multicolumn{2}{|c|}{ reference } \\
\hline & Completed & 219 & 23.74 & 1.22 & 0.3 & 1.07 & 0.8 & 1.07 & 0.8 \\
\hline & \multicolumn{9}{|l|}{ Occupation } \\
\hline & Professional & 105 & 22.86 & \multicolumn{2}{|c|}{ reference } & \multicolumn{2}{|c|}{ reference } & \multicolumn{2}{|c|}{ reference } \\
\hline & Associate professional & 53 & 22.64 & 0.99 & 1.0 & 0.96 & 0.9 & 0.96 & 0.9 \\
\hline & Manager & 55 & 23.64 & 1.04 & 0.9 & 1.04 & 0.9 & 1.04 & 0.9 \\
\hline & Clerical or Service & 48 & 27.08 & 1.25 & 0.4 & 1.51 & 0.2 & 1.51 & 0.2 \\
\hline & \multicolumn{9}{|l|}{ Marital Status } \\
\hline & Married/de facto & 200 & 23 & \multicolumn{2}{|c|}{ reference } & & & & \\
\hline & Widowed/separated/divorced & 26 & 23.08 & 1.00 & 1.0 & 1.34 & 0.7 & 1.34 & 0.7 \\
\hline & Never married & 60 & 23.33 & 1.02 & 1.0 & 0.71 & 0.4 & 0.71 & 0.4 \\
\hline & BASELINE MEASURES & & & & & & & & \\
\hline 3 & Prior GCC $^{\circledR}$ Participation ${ }^{\complement}$ & 286 & 22.54 & 0.96 & 0.9 & 0.88 & 0.6 & 1.13 & 0.6 \\
\hline & Reasons for Participation ${ }^{c}$ & & & & & & & & \\
\hline & Health & 286 & 21.92 & 0.76 & 0.4 & 1.22 & 0.6 & 1.22 & 0.6 \\
\hline & To look my best & 286 & 19.23 & 0.48 & $<0.001$ & 0.38 & 0.004 & 0.38 & 0.004 \\
\hline & Fitness & 286 & 20.57 & 0.61 & 0.2 & 0.93 & 0.9 & 0.93 & 0.9 \\
\hline & Colleagues & 286 & 23.9 & 1.11 & 0.7 & 0.89 & 0.7 & 0.89 & 0.7 \\
\hline & Friends or family & 286 & 14.29 & 0.55 & 0.6 & 0.69 & 0.8 & 0.69 & 0.8 \\
\hline & Behavioural Measures & & & & & & & & \\
\hline 4 & Fruit Intake & & & & & & & & \\
\hline & Not meeting guidelines & 188 & 19.15 & & arence & & & & \\
\hline & Meeting guidelines & 98 & 30.61 & 1.86 & 0.028 & 2.42 & $<0.001$ & 3.05 & 0.001 \\
\hline & Vegetable Intake & & & & & & & & \\
\hline & Not meeting guidelines & 244 & 23.36 & & arence & & & & \\
\hline & Meeting guidelines & 42 & 21.43 & 0.89 & 0.8 & 0.62 & 0.4 & 0.61 & 0.4 \\
\hline
\end{tabular}


Takeaway Dinner

Once or less per month

About once a week

More than once a week Alcoho

Not meeting guidelines Meeting guidelines

Tobacco

Smoker

Non-smoker

Physical Activity

Not meeting guidelines

Meeting guidelines

Sitting Time (hrs per day)

Weekday

Weekend

Psychosocial Measures

5 Physical Functioning (SF12)

Mental Functioning (SF12)

Physical Measures

6 Blood Pressure $(\mathrm{mmHg})$

Systolic

Diastolic

Not meeting guidelines

Meeting guidelines

Heart rate (Mean, SD)

Weight (Mean, SD)

Body Mass Index

Body Mass Index

Not meeting guidelines

Meeting guidelines

Waist circumference

Biomedical Measures (fasting) ${ }^{d}$

Total Cholesterol

Total Cholesterol

Not meeting guidelines

Meeting guidelines
Waist circumference

\subsection{5}

24.79

27.03

24.53

21.26

21.88

23.23

20.79

26.85

286

282

282

56

217

273

288

238

288

Glucose reference

$\begin{array}{lll}1.28 & 0.4 & 1.35\end{array}$

$1.44 \quad 0.3$

$$
1.44
$$

reference

$$
0.83 \quad 0.6
$$

0.3

0.64

reference

$1.08 \quad 0.8$

0.71

0.5

0.61

reference

$1.40 \quad 0.002$

1.42

0.1

1.48

$0.94 \quad 0.1$

$0.80 \quad \mathbf{0 . 0 2 5}$

0.99

0.9

1.00

$1.07<0.001$

1.08

0.053

0.023

$1.00 \quad 0.6$

$<0.001$

0.004

$0.97<0.001$

$0.95<0.001$

reference

$4.74<0.001$

$0.99 \quad 0.8$

$0.94<0.001$

$0.64<0.001$

reference

$8.42<0.001$

$0.91<0.001$

$0.60 \quad \mathbf{0 . 0 0 1}$

0.66

0.018

0.63

$<0.001$
$2.11 \quad \mathbf{0 . 0 4 0}$ 


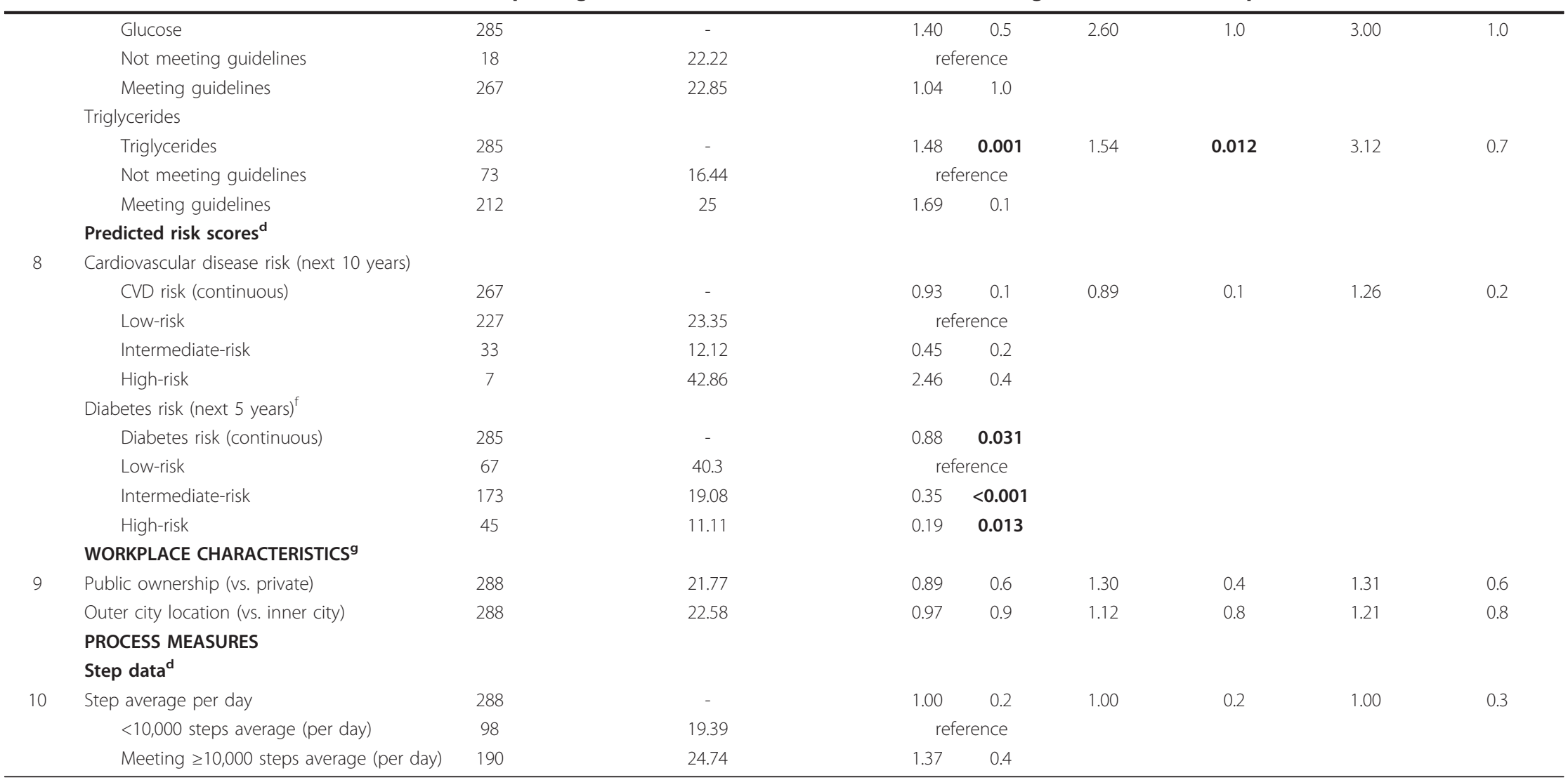

a Associated risk-factor subgroups adjusted only for baseline demographic confounders
b Associated risk-factor subgroups adjusted for all potential predictors within the analysis Group and above
' The reference group for this binary variable is 'no'. The reference group data is not shown.

${ }^{\mathrm{d}}$ Continuous variables chosen over categorical variables

${ }^{\mathrm{e}}$ Excluded due to the inclusion of body mass index (BMI)

${ }^{f}$ Excluded from model as baseline WC was not considered as a predictor.

${ }^{g}$ The reference group for this binary variable is shown in brackets. The reference group data is not shown.

Note: For participants not meeting WC guidelines at baseline 
compared to participants who had not completed tertiary education at baseline. For every millimole per litre less of total cholesterol at baseline, a $0.9 \mathrm{~cm}$ greater loss in WC at four-months was observed.

\section{Predictors of improving waist circumference to meet low- risk guidelines at four-months}

Between baseline and four-months, $22.9 \%$ of participants who had high-risk WC at baseline improved their WC enough to meet low-risk guidelines at four-months, Table 5. Through univariable analysis, new baseline variables associated with reducing WC to meet low-risk guidelines at four-months, included not participating in the program for appearance reasons, meeting fruit intake guidelines, meeting physical activity guidelines, less weekend sitting time, higher physical functioning, lower systolic and diastolic blood pressure, meeting guidelines for blood pressure, lower baseline weight, lower BMI, meeting BMI guidelines, smaller WC, lower total cholesterol, meeting total cholesterol guidelines, more elevated triglycerides and higher diabetes risk, Table 5.

Although all participants in this sub-analysis had highrisk WC at baseline, a few were meeting guidelines for body composition when assessed by BMI. Within the high-risk WC group at baseline, participants who were meeting guidelines for BMI at baseline responded positively to the program and had 8.4 increased odds of improving their $\mathrm{WC}$ to meeting guidelines at four-months than participants not meeting baseline BMI guidelines, $\mathrm{p}<$ 0.001 . Participants with low baseline diabetes type- 2 risk had 5.4 increased odds of improving their WC to meeting guidelines at four-months than participants who were at high baseline diabetes risk, $\mathrm{p}=0.013$.

Within the high-risk baseline WC group, for every year increase in age at baseline, it was $3 \%$ less likely that the participant would improve their WC to meet low-risk guidelines at four-months. Employees participating in the program for reasons other than appearance had 2.6 increased odds of improving their WC to meet guidelines than employees participating for other reasons. Participants eating two or more pieces of fruit per day at baseline were 3.1 times more likely to improve their WC to meet guidelines than participants eating less than two pieces per day. For every hour decrease of weekend sitting time at baseline, it was 33\% more likely that the participant would improve their WC to meet guidelines at four-months. For every BMI unit decrease at baseline, it was twice as likely that the participant would improve their WC to meet guidelines at four-months.

\section{Discussion}

In this study analysing variables associated with waist circumference (WC) change following participation in a four-month, pedometer-based, workplace health program, employees with a high-risk WC at baseline experienced the greatest improvements in WC. Strong predictors of improved WC during the program for all employees and employees with high-risk baseline WC were having completed tertiary education, undertaking less baseline weekend sitting time and having lower total cholesterol at baseline. An additional predictor of improvement in WC for all employees was not consuming more than two standard alcoholic beverages in one occasion during the twelve months prior to baseline. Unique baseline predictors were identified for improving WC to meet guidelines at fourmonths and these included participating for reasons other than appearance, eating at least two serves of fruit per day, higher physical functioning and lower BMI.

Our finding that employees with a high-risk WC at baseline experienced the greatest improvements in WC confirms findings from a similar workplace health program evaluation [14]. However, the relationship between baseline WC and WC change seems to be contradictory to the relationships observed with the other predictors, which mainly indicated better health. The finding that employees with larger $\mathrm{WC}$ at baseline improved the most during the program may be because they had the greatest opportunity to improve. In addition, some unique variables associated with improved WC that indicated better health were restricted to the high-risk baseline WC group.

The finding that employees with better health benefited the most from the program may indicate that healthier employees may find it easier to make the small changes required for a visible outcome. Whilst others, who need to make a greater change, may need additional support or motivation that may not be available through a workplace health program alone. However, a horse-racing effect [26] may be also present and as we did not assess behavioural change status, employees who are defined as 'healthier' at baseline may actually have recently made positive changes and be on a pathway to reducing their health risks. Hence, the workplace health program could be supporting employees to continue making the healthier behavioural changes. An alternate theory is that the markers of better health may be indicators of better socioeconomic status. However, when several socio-economic confounders were included in the model, only adequate fruit intake in the high-risk baseline WC sub-analysis became non-significant.

Regardless of the reasoning for the healthier employees responding better to the program, the result highlights a possible need for these programs to respond to the enrolees and encourage change based on their individual baseline characteristics. Program initiators may need to think innovatively about how to further promote change in employees with multiple baseline risk factors. 
The strengths of this evaluation included the range and quality of measurements, the large sample size and the variety of sedentary occupations within the sample [15]. The main limitation is the lack of assessment and evaluation of program and workplace characteristics. We recommend that future health programs not only evaluate a wide range of participant predictors (behavioural, anthropological and biological), but also the program and workplace characteristics as predictors. Another limitation is that the potential selection bias associated with workplace recruitment, individual recruitment and participant retention $[9,15]$. Workplaces electing evaluation may have been more motivated as they conscripted to the $\mathrm{GCC}^{\circledR} 2008$ early, study participants may have been more motivated and/or have better baseline health and returning study participants had healthier baseline measures $[9,15,27,28]$. A healthier, more motivated cohort would be more likely to comply with the health program (overestimating the health benefits) but a greater proportion of a healthier cohort would already be meeting health guidelines at baseline (underestimating the general health benefits of participation due to ceiling effects) [9]. However, selection bias is unlikely to substantially affect the interrelationships between predictors and WC change. A potential limitation is not having an explicit measure of the potential regression to the mean effect for waist circumference. However, as waist circumference decreased over time in both the low and high baseline risk groups there was no evidence of the regression to the mean effect in this sample.

\section{Conclusions}

While employees with high-risk WC at baseline experienced the greatest improvements in WC, the other predictors of WC improvement were generally indicators of better health at baseline. These results indicate that employees who started with better health, potentially due to lifestyle or recent behavioural changes, were more likely to respond positively to the program. The results from this paper can be used to inform employees during recruitment for workplace physical activity programs that there are benefits for participants who are in the healthier spectrum, as well as employees who have a higher WC risk. However, we suggest that these programs may need to promote additional support and motivation for participants who are at greatest health risk. We encourage future health program initiators to think innovatively about how to encourage all enrolees along the health spectrum to achieve clinically relevant improvement.

\section{Authors' contributions}

RFP and AP undertook the study design and oversaw the data collection for the project. RFP takes responsibility for the integrity of the data and the accuracy of the data analysis. RFP, RW and AP contributed to the statistical data analysis. RFP, RW, HW, KB \& AP contributed to the critical interpretation of the data. All authors contributed to the final version of the paper and have read, as well as, approved the final manuscript.

\section{Competing interests}

This data was collected as part of the Global Corporate Challenge ${ }^{{ }_{\oplus}}$ Evaluation Study conducted through Monash University. We would like to acknowledge Maximilian de Courten and Margaret Brand for their assistance during this process. We would like to acknowledge the Australian Research Council (ARC) and the Foundation for Chronic Disease Prevention ${ }^{\text {TM }}$ in the Workplace, which is associated with the Global Corporate Challenge ${ }^{{ }_{\oplus}}$, for partially funding this study. The study design; analysis and interpretation of data; the writing of the manuscript; and the decision to submit the manuscript for publication were solely at the discretion of the Monash researchers, independent of GCC ${ }^{\mathbb{B}_{\circledast}}$ or The Foundation's involvement. RFP, RW, HW, KB \& AP are affiliated with Monash University. RFP is supported by an Australian Postgraduate Award and a Monash Departmental Scholarship. AP is funded by a VicHealth Public Health Fellowship. The authors declare that they have no further competing interests.

Received: 16 February 2011 Accepted: 25 October 2011

Published: 25 October 2011

ReferencesWorld Health Organization \& World Economic Forum: Preventing Noncommunicable Diseases in the Workplace through Diet and Physical Activity: WHO/World Economic Forum Report of a Joint Event. Geneva: World Health Organization; 2008.

2. Goetzel RZ, Ozminkowski RJ: The health and cost benefits of work site health-promotion programs. Annu Rev Public Health 2008, 29:303-323.

3. Conn VS, Hafdahl AR, Cooper PS, Brown LM, Lusk SL: Meta-analysis of workplace physical activity interventions. Am J Prev Med 2009, 37(4):330-339.

4. Eaton CB, Menard LM: A systematic review of physical activity promotion in primary care office settings. Br J Sports Med 1998, 32(1):11-16.

5. Katz DL, O'Connell M, Yeh MC, Nawaz H, Njike V, Anderson LM, Cory S, Dietz W: Public health strategies for preventing and controlling overweight and obesity in school and worksite settings: a report on recommendations of the Task Force on Community Preventive Services. MMWR Recomm Rep 2005, 54(RR-10):1-12.

6. Task Force on Community Preventive Services: Recommendations for worksite-based interventions to improve workers' health. Am J Prev Med 2010, 38(2 Suppl):S232-236.

7. Robroek SJ, van Lenthe FJ, van Empelen P, Burdorf A: Determinants of participation in worksite health promotion programmes: a systematic review. Int J Behav Nutr Phys Act 2009, 6:26.

8. Soler RE, Leeks KD, Razi S, Hopkins DP, Griffith M, Aten A, Chattopadhyay SK, Smith SC, Habarta N, Goetzel RZ, et al: A systematic review of selected interventions for worksite health promotion. The assessment of health risks with feedback. Am J Prev Med 2010, 38(2 Suppl):S237-262.

9. Freak-Poli R, Wolfe R, Backholer K, de Courten M, Peeters A: Impact of a pedometer-based workplace health program on cardiovascular and diabetes risk profile. Prev Med 2011, 53(3):162-171.

10. Kwak L, Kremers SP, Candel MJ, Visscher TL, Brug J, van Baak MA: Changes in skinfold thickness and waist circumference after 12 and 24 months resulting from the NHF-NRG In Balance-project. Int I Behav Nutr Phys Act 2010, 7:26.

11. Feller $\mathrm{S}$, Boeing $H$, Pischon T: Body mass index, waist circumference, and the risk of type 2 diabetes mellitus: implications for routine clinical practice. Dtsch Arztebl Int 2010, 107(26):470-476.

12. Wang Z, Hoy WE: Body size measurements as predictors of type 2 diabetes in Aboriginal people. Int J Obes Relat Metab Disord 2004, 28(12):1580-1584.

13. Wang Z, Hoy WE: Waist circumference, body mass index, hip circumference and waist-to-hip ratio as predictors of cardiovascular disease in Aboriginal people. Eur J Clin Nutr 2004, 58(6):888-893.

14. Chan CB, Ryan DA, Tudor-Locke C: Health benefits of a pedometer-based physical activity intervention in sedentary workers. Prev Med 2004, 39(6):1215-1222

15. Freak-Poli R, Wolfe R, Peeters A: Risk of cardiovascular disease and diabetes in a working population with sedentary occupations. J Occup Environ Med 2010, 52(11):1132-1137. 
16. World Health Organisation: WHO STEPS instrument. Geneva: Department of Chronic Diseases and Health Promotion, World Health Organisation; 2002.

17. Dunstan DW, Zimmet PZ, Welborn TA, Cameron AJ, Shaw J, de Courten M, Jolley D, McCarty DJ, Australian Diabetes O, Lifestyle S: The Australian Diabetes, Obesity and Lifestyle Study (AusDiab)-methods and response rates. Diabetes Res Clin Pract 2002, 57(2):119-129.

18. Giles GG, English DR: The Melbourne Collaborative Cohort Study. IARC Sci Publ 2002, 156:69-70

19. Ball K, Brown W, Crawford D: Who does not gain weight? Prevalence and predictors of weight maintenance in young women. Int $J$ Obes Relat Metab Disord 2002, 26(12):1570-1578.

20. Reduce Your Risk: New National Guidelines for Alcohol Consumption. 2011 [http://www.health.gov.au/internet/alcohol/publishing.nsf/Content/ 36E6FEE732C8DF1BCA25767200769CD8/\$File/adult.pdf], Accessed Feb.

21. Baik I, Ascherio A, Rimm EB, Giovannucci E, Spiegelman D, Stampfer MJ, Willett WC: Adiposity and mortality in men. American journal of epidemiology 2000, 152(3):264-271.

22. Visscher TL, Seidell JC, Molarius A, van der Kuip D, Hofman A, Witteman JC: A comparison of body mass index, waist-hip ratio and waist circumference as predictors of all-cause mortality among the elderly: the Rotterdam study. Int J Obes Relat Metab Disord 2001, 25(11):1730-1735.

23. Jacobs EJ, Newton CC, Wang Y, Patel AV, McCullough ML, Campbell PT, Thun MJ, Gapstur SM: Waist circumference and all-cause mortality in a large US cohort. Arch Intern Med 2010, 170(15):1293-1301.

24. Testa G, Cacciatore F, Galizia G, Della-Morte D, Mazzella F, Langellotto A, Russo S, Gargiulo G, De Santis D, Ferrara N, et al: Waist Circumference but Not Body Mass Index Predicts Long-Term Mortality in Elderly Subjects with Chronic Heart Failure. J Am Geriatr Soc 2010

25. Seidell JC: Waist circumference and waist/hip ratio in relation to all-cause mortality, cancer and sleep apnea. Eur J Clin Nutr 2010, 64(1):35-41.

26. Glymour MM, Weuve J, Berkman LF, Kawachi I, Robins JM: When is baseline adjustment useful in analyses of change? An example with education and cognitive change. American journal of epidemiology 2005, 162(3):267-278.

27. Richmond R, Wodak A, Bourne S, Heather N: Screening for unhealthy lifestyle factors in the workplace. Aust N Z J Public Health 1998, 22(3 Suppl):324-331.

28. Sorensen G, Stoddard A, Ockene JK, Hunt MK, Youngstrom R: Worker participation in an integrated health promotion/health protection program: results from the WellWorks project. Health Educ Q 1996, 23(2):191-203.

29. Australian Institute of Health and Welfare (AlHW): Australia's health 2008. Cat. no. AUS 99. Canberra: AlHW; 2008.

30. DHAC (Commonwealth Government Department of Health and Aged Care): National physical activity guidelines for Australians. Canberra: DHAC; 1999.

31. Royal Australian College of General Practitioners. National Preventive and Community Medicine Committee. Guidelines for preventive activities in general practice. 7 edition. Victoria: The Royal Australian College of General Practitioners; 2009.

32. National Health and Medical Research Council (NHMRC): Food for health: Dietary Guidelines for all Australians. Canberra: NHMRC; 2003.

33. Huang N: Guide to management of hypertension. Australian Prescriber 2006, 31(6):150-153.

34. National Health and Medical Research Council (NHMRC): Overweight and obesity in adults: a guide for general practitioners Canberra: NHMRC; 2003.

35. World Health Organization (WHO): Obesity: preventing and managing the global epidemic. Who Technical Report Series 894 WHO Consultation on Obesity. Geneva: WHO; 2004.

36. National Health and Medical Research Council (NHMRC): Clinical practice guidelines for the management of overweight and obesity in adults Canberra: NHMRC; 2003.

37. High blood cholesterol. [http://www.aihw.gov.au/riskfactors/cholesterol. $\mathrm{cfm}]$.

38. National Heart Foundation of Australia and the Cardiac Society of Australia and New Zealand: Position Statement on Lipid Management-2005 Heart Lung and Circulation. 2005, 14:275-291.

39. Baker IDI Heart and Diabetes Institute on behalf of the Australian State and Territory Governments as part of the COAG initiative to reduce the risk of type 2 diabetes: The Australian Type 2 Diabetes Risk Assessment Tool
(AUSDRISK). Department of Health and Ageing, Australian Government; 2008

40. Chen L, Magliano DJ, Balkau B, Colagiuri S, Zimmet PZ, Tonkin AM, Mitchell P, Phillips PJ, Shaw JE: AUSDRISK: an Australian Type 2 Diabetes Risk Assessment Tool based on demographic, lifestyle and simple anthropometric measures. Med J Aust 2010, 192(4):197-202.

41. D'Agostino RB Sr, Vasan RS, Pencina MJ, Wolf PA, Cobain M, Massaro JM Kannel WB: General cardiovascular risk profile for use in primary care: the Framingham Heart Study. Circulation 2008, 117(6):743-753.

42. National Vascular Disease Prevention Alliance: Absolute cardiovascular disease risk. Technical report: review of the evidence and evidencebased recommendations for practice. 2009.

43. Guidelines for the assessment of absolute cardiovascular disease risk. [http://www.heartfoundation.org.au/SiteCollectionDocuments/absolute-riskassessement.pdf].

\section{Pre-publication history}

The pre-publication history for this paper can be accessed here: http://www.biomedcentral.com/1471-2458/11/824/prepub

\section{doi:10.1186/1471-2458-11-824}

Cite this article as: Freak-Poli et al:: Participant characteristics associated with greater reductions in waist circumference during a four-month, pedometer-based, workplace health program. BMC Public Health 2011 11:824.

\section{Submit your next manuscript to BioMed Central and take full advantage of:}

- Convenient online submission

- Thorough peer review

- No space constraints or color figure charges

- Immediate publication on acceptance

- Inclusion in PubMed, CAS, Scopus and Google Scholar

- Research which is freely available for redistribution

Submit your manuscript at www.biomedcentral.com/submit
C Biomed Central 